\title{
ESTUDO DO EQUILÍBRIO LÍQUIDO-LÍQUIDO PARA O SISTEMA CONTENDO BIODIESEL METÍLICO DE COCO BABAÇU, METANOL E GLICEROL
}

\author{
R. S. ALVES ${ }^{1}$, L. C. LOPES $^{1}$, A. M. M. BESSA ${ }^{1}$, R. S. de S. AGUIAR ${ }^{1}$ e H. B. de \\ SANT' $^{\prime}$ ANA $^{1}$ \\ ${ }^{1}$ Universidade Federal do Ceará, Departamento de Engenharia Química \\ E-mail para contato: raissa.s.alves@gmail.com
}

\begin{abstract}
RESUMO - O biodiesel é um combustível derivado de óleos vegetais e animais, desenvolvido para o uso em substituição ao diesel derivado do petróleo. Consiste em uma mistura de ésteres advindos principalmente da reação de transesterificação entre triglicerídeos e/ou ácidos graxos livres com álcoois de cadeira curta, por catálise. Essa reação produz glicerol como subproduto, que deve ser separado do biodiesel juntamente com as impurezas presentes. Portanto, o estudo do equilíbrio liquido-liquido (ELL) dos compostos envolvidos na produção do biodiesel é de importância para o fornecimento de meios para a otimização dos processos de separação. O principal objetivo deste trabalho foi determinar dados de ELL para o sistema ternário biodiesel de coco babaçu + metanol + glicerol, a $20{ }^{\circ} \mathrm{C}$ e $40{ }^{\circ} \mathrm{C}$ e pressão atmosférica. Onde se observou que influência da temperatura no comportamento de fases pode ser negligenciável. Os dados de ELL foram determinados através de técnicas titulométrica e densimétrica. A consistência termodinâmica dos dados foi verificada pela correlação de Hand. Os modelos NRTL, UNIQUAC e UNIFAC foram utilizados para correlacionar os dados de equilíbrio para coeficientes de atividade.
\end{abstract}

\section{INTRODUÇÃO}

Nas últimas décadas a sociedade tem buscado diminuir sua dependência energética de fontes fósseis devido à possibilidade de escassez (fonte não renovável), ao alto custo de extração e refino e à liberação de enxofre em sua queima, prejudicando o meio ambiente. Nessa conjuntura o biodiesel surge como fonte alternativa ao diesel derivado do petróleo principalmente por suas características físico-químicas, e contribuição nos âmbitos social e econômico. Além disso, pode ser aplicado nos motores atuais sem modificações mecânicas. Biodiesel é definido como uma mistura de ésteres de óleos vegetais e/ou animais que pode ser obtido através de craqueamento catalítico ou reação de transesterificação. A reação de transesterificação com metanol, por via catalítica homogênea, é o processo mais simples e comumente utilizado para a conversão de óleos e gorduras em ésteres de interesse (Knothe et al., 2008). A composição da mistura de ésteres é determinada por sua matéria-prima de origem. As matérias-primas devem ser compostas prioritariamente por triglicerídeos e são representadas principalmente por óleos vegetais como soja, milho, algodão, babaçu, entre outros. Embora na literatura já existam trabalhos abrangendo o estudo de óleos vegetais, o biodiesel advindo do coco babaçu não possui demasiados estudos. Além disto, neste trabalho, 
a escolha do babaçu como matéria-prima deu-se por características geoeconômicas e tipicidade no nordeste brasileiro, cujo potencial econômico ainda é pouco explorado (Lima et al., 2007). Após o término da reação de transesterificação, os produtos (biodiesel e glicerol) são separados por decantação e o catalisador restante, juntamente com o álcool em excesso, é extraído por lavagens com água. Neste contexto, conhecer as condições de equilíbrio líquidolíquido envolvidas na produção desse biodiesel é de importância para o fornecimento de meios para a otimização dos processos de separação e realização de simulações computacionais.

\section{MATERIAIS E MÉTODOS}

O biodiesel utilizado no presente trabalho foi produzido no Laboratório de FísicoQuímica Aplicada - Universidade Federal do Ceará, por meio de transesterificação alcalina. A composição do biodiesel foi analisada por cromatografia gasosa com detector de ionização de chama (FID). O Glicerol foi fornecido pela Vetec, com grau de pureza superior a 99,5\%, já o metanol fornecido pela Synth, com grau de pureza superior a 99,8\%; ambos sem processos extras de purificação

Os dados experimentais foram determinados através do uso de células de vidro encamisadas, em conformidade com Stragevitch (1997). Essas são providas de coletores laterais vedados por septos que permitem a coleta de amostras de cada fase, por seringas, sem que haja perturbação do equilíbrio. Além disso, a temperatura do sistema mantém-se constante e pode ser controlada por um banho termostático da Tecnal, modelo TE-184, neste caso à $20^{\circ} \mathrm{C}$ e $40^{\circ} \mathrm{C}$. De acordo com Gonzalez et al. (1986) as curvas binodais ou curvas de equilíbrio dos sistemas ternários, podem ser determinadas pelo método do ponto de turbidez, ou seja, para construção dessas partiu-se de uma mistura binária, de massas conhecidas, que foi titulada por intermédio de um terceiro componente gota a gota sob agitação, em um agitador magnético da Tecnal modelo TE-0851 com auxílio de uma barra magnética de Teflon, até o aparecimento e permanência de turbidez visual por pelo menos 30 segundos. A massa do componente titulante é determinada por gravimetria, antes e depois da titulação, com o auxílio de uma balança analítica da marca Shimadzu, modelo AY-220, com precisão de $+/-0,0001 \mathrm{~g}$. A amostra de pelo menos $5 \mathrm{~mL}$ é retirada do ponto de turbidez para análise de densidade, através de um densímetro da marca Anton Paar, modelo DSA-5000. As duas fases de estudo foram: Fase I (rica em biodiesel), construída a partir de uma mistura binária de biodiesel metílico de coco babaçu e metanol, titulada com glicerol; e, Fase II (rica em glicerol), construída a partir de uma mistura binária de glicerol e metanol, titulada com biodiesel. Conhecendo a curva binodal (de solubilidade), ou seja, a delimitação da região homogênea, foram estimados valores de composição global dentro desta curva e pesados nas células de equilíbrio. As misturas foram agitadas por $3 \mathrm{~h}$ e permaneceram em repouso por 14 $\mathrm{h}$, onde a formação de uma interface bem definida entre as duas fases foi observada. Para cada fase, dados de densidade foram determinados, conhecendo-se, assim, suas composições.

\section{MODELAGEM TERMODINÂMICA}

As modelagens desenvolvidas neste trabalho utilizaram o programa Visual Studio 2013 implementando os modelos NRTL (Renon et al., 1968), que se baseia no conceito de composição local; UNIQUAC (Abrams et al., 1975), com base teórica na mecânica estatística 


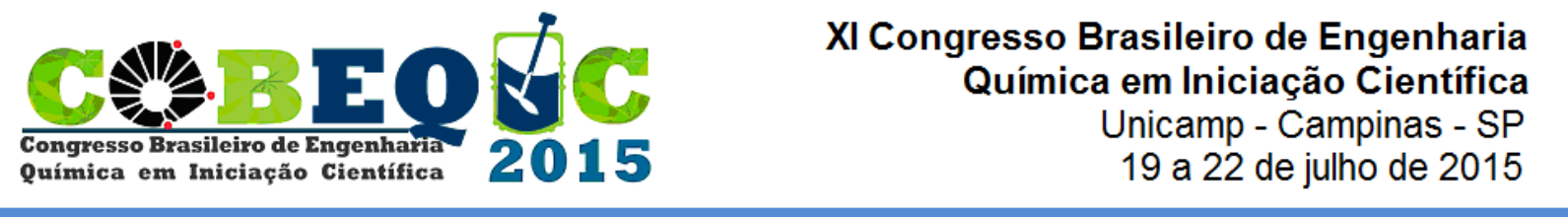

e UNIFAC (Fredenslund et al., 1977), com base na contribuição de grupo. Os parâmetros UNIFAC utilizados se basearam no desenvolvimento de Magnussen (1981) de interação de grupo para os sistemas de ELL. Neste trabalho o procedimento é baseado no método Simplex modificado (Nelder et al., 1965) para minimizar uma função objetivo (eq. 1) de composições dos componentes, em que $\mathrm{D}$ representa o número de conjunto de dados e $\mathrm{N}$ e $\mathrm{M}$, respectivamente, o número de componentes e linhas de amarração em cada conjunto de dados. Já os sobrescritos I e II referem-se às duas fases em equilíbrio; e os sobrescritos 'exp' e 'calc' referem-se às composições, experimentais ou calculadas, utilizando-se o parâmetro estatístico do desvio médio quadrático.

$$
\text { F.O. }=\sum_{k}^{D} \sum_{j}^{M} \sum_{i}^{N-1}\left(x_{i j k}^{I, \exp }-x_{i j k}^{I, \text { calc }}\right)+\left(x_{i j k}^{I I, \exp }-x_{i j k}^{I I, \text { calc }}\right)
$$

\section{RESULTADOS E DISCUSSÕES}

\subsection{Curvas de Calibração}

De acordo com a metodologia adotada neste trabalho (densimetria e titulometria), previamente certificada pela reprodução dos dados de Zhang e Hill (1991), dados das frações mássicas e de densidade foram utilizados para a construção das curvas de solubilidade e são apresentados abaixo na Tabela 1.

Tabela 1 - Dados de densidade e composição das curvas de calibração para o sistema biodiesel (1) + metanol (2) + glicerol (3) a 20 e $40{ }^{\circ} \mathrm{C}$

\begin{tabular}{|c|c|c|c|c|c|c|c|}
\hline \multicolumn{4}{|c|}{ Biodiesel de babaçu + metanol + glicerol a $20^{\circ} \mathrm{C}$} & \multicolumn{4}{|c|}{ Biodiesel de babaçu + metanol + glicerol a $40{ }^{\circ} \mathrm{C}$} \\
\hline $\mathrm{W}_{1}$ & $\mathrm{w}_{2}$ & $\mathrm{w}_{3}$ & $\begin{array}{c}\text { Densidade } \\
\left(\mathrm{g} / \mathrm{cm}^{3}\right)\end{array}$ & $\mathrm{w}_{1}$ & $\mathrm{w}_{2}$ & $\mathrm{w}_{3}$ & $\begin{array}{c}\text { Densidade } \\
\left(\mathrm{g} / \mathrm{cm}^{3}\right)\end{array}$ \\
\hline \multicolumn{8}{|c|}{ Fase rica em biodiesel de babaçu } \\
\hline 0,2342 & 0,6990 & 0,0669 & 0,833398 & 0,8558 & 0,1305 & 0,0137 & 0,851757 \\
\hline 0,3406 & 0,5945 & 0,0649 & 0,841116 & 0,7309 & 0,2433 & 0,0257 & 0,845886 \\
\hline 0,4744 & 0,4782 & 0,0474 & 0,84575 & 0,4632 & 0,4637 & 0,0731 & 0,839447 \\
\hline 0,5974 & 0,3624 & 0,0402 & 0,85289 & 0,3501 & 0,5808 & 0,0691 & 0,830052 \\
\hline 0,7288 & 0,2443 & 0,0270 & 0,85893 & 0,2292 & 0,6848 & 0,0860 & 0,826088 \\
\hline 0,8540 & 0,1285 & 0,0175 & 0,865459 & 0,9918 & 0,0000 & 0,0082 & 0,860259 \\
\hline 0,9970 & 0,0000 & 0,0030 & 0,874133 & 0,6577 & 0,3011 & 0,0412 & 0,844777 \\
\hline- & - & - & - & 0,7947 & 0,1874 & 0,0179 & 0,850221 \\
\hline \multicolumn{8}{|c|}{ Fase rica em glicerol } \\
\hline 0,0521 & 0,1195 & 0,8284 & 1,183003 & 0,0073 & 0,1259 & 0,8668 & 1,171024 \\
\hline 0,0207 & 0,2469 & 0,7325 & 1,111606 & 0,0160 & 0,2481 & 0,7359 & 1,100474 \\
\hline 0,0318 & 0,3649 & 0,6033 & 1,048377 & 0,0139 & 0,3729 & 0,6132 & 1,039470 \\
\hline 0,0233 & 0,5005 & 0,4763 & 0,986658 & 0,0121 & 0,4936 & 0,4944 & 0,986140 \\
\hline 0,0289 & 0,6086 & 0,3625 & 0,935172 & 0,0199 & 0,6140 & 0,3661 & 0,923126 \\
\hline 0,0395 & 0,7215 & 0,2390 & 0,884871 & 0,0618 & 0,7035 & 0,2347 & 0,871939 \\
\hline 0,0487 & 0,7558 & 0,1956 & 0,868304 & 0,0249 & 0,0000 & 0,9751 & 1,242297 \\
\hline 0,1088 & 0,7802 & 0,1110 & 0,839971 & 0,1040 & 0,7396 & 0,1564 & 0,837641 \\
\hline 0,0463 & 0,0000 & 0,9537 & 1,25729 & - & - & - & - \\
\hline
\end{tabular}


Observou-se que para os sistemas estudados, em ambas as temperaturas, houve um aumento na densidade de acordo com o aumento da fração mássica do componente rico da fase. Este resultado reflete a diferença de massa molar entre os componentes do sistema. A partir dos dados da Tabela 1, curvas de calibração foram construídas por componente e fase gerando equações de ajuste, por intermédio da qual as frações mássicas dos componentes podem ser determinado via balanço de massa.

\subsection{Linhas de Amarração}

As composições das misturas globais e de suas respectivas fases em equilíbrio, obtidas através das equações de ajuste, são apresentadas na Tabela 2. A partir dos apresentados nas Tabelas 1 e 2, pode-se obter o gráfico ternário comparativo entre as temperaturas de $20^{\circ} \mathrm{C}$ e $40^{\circ} \mathrm{C}$ pode ser gerado (Figura 1).

Tabela 2 - Dados de fração mássica das linhas de amarração para o sistema biodiesel de babaçu (1) + metanol (2) + glicerol a $20^{\circ} \mathrm{C}$ e $40{ }^{\circ} \mathrm{C}$

\begin{tabular}{|c|c|c|c|c|c|c|c|c|}
\hline \multicolumn{3}{|c|}{ Composição global } & \multicolumn{3}{|c|}{ Fase rica em biodiesel } & \multicolumn{3}{|c|}{ Fase rica em glicerol } \\
\hline $\mathrm{w}_{1}$ & $\mathrm{w}_{2}$ & $\mathrm{~W}_{3}$ & $\mathrm{w}_{1}$ & $\mathrm{~W}_{2}$ & $\mathrm{~W}_{3}$ & $\mathrm{w}_{1}$ & $\mathrm{w}_{2}$ & $\mathrm{~W}_{3}$ \\
\hline \multicolumn{9}{|c|}{ Biodiesel babaçu (1) + metanol (2) + glicerol (3) a $20^{\circ} \mathrm{C}$} \\
\hline 0,375103 & 0,248983 & 0,375914 & 0,8969 & 0,0906 & 0,0125 & 0,0240 & 0,3549 & 0,6211 \\
\hline 0,343332 & 0,320878 & 0,335789 & 0,8579 & 0,1260 & 0,0160 & 0,0238 & 0,4398 & 0,5365 \\
\hline 0,412643 & 0,174227 & 0,413131 & 0,9357 & 0,0554 & 0,0089 & 0,0278 & 0,2606 & 0,7116 \\
\hline 0,44159 & 0,116785 & 0,441625 & 0,9611 & 0,0323 & 0,0066 & 0,0337 & 0,1778 & 0,7885 \\
\hline 0,314557 & 0,37111 & 0,314333 & 0,8600 & 0,1242 & 0,0158 & 0,0256 & 0,4961 & 0,4783 \\
\hline 0,292087 & 0,41935 & 0,288563 & 0,8419 & 0,1406 & 0,0175 & 0,0292 & 0,5520 & 0,4188 \\
\hline \multicolumn{9}{|c|}{ Biodiesel babaçu (1) + metanol (2) + glicerol (3) a $40^{\circ} \mathrm{C}$} \\
\hline 0,378788 & 0,246996 & 0,374216 & 0,9002 & 0,0873 & 0,0873 & 0,0070 & 0,3741 & 0,6189 \\
\hline 0,343787 & 0,320282 & 0,335931 & 0,8759 & 0,1102 & 0,1102 & 0,0139 & 0,4612 & 0,5249 \\
\hline 0,415671 & 0,171463 & 0,412866 & 0,9082 & 0,0798 & 0,0798 & 0,0050 & 0,2763 & 0,7186 \\
\hline 0,440702 & 0,117336 & 0,441961 & 0,9498 & 0,0407 & 0,0407 & 0,0079 & 0,1880 & 0,8041 \\
\hline 0,315388 & 0,372409 & 0,312202 & 0,8583 & 0,1267 & 0,1267 & 0,0217 & 0,5192 & 0,4590 \\
\hline 0,290322 & 0,418658 & 0,291021 & 0,8387 & 0,1449 & 0,1449 & 0,0293 & 0,5617 & 0,4090 \\
\hline
\end{tabular}

Figura 1 - Dados de equilíbrio líquido-líquido para o sistema biodiesel de coco babaçu + metanol + glicerol: $20^{\circ} \mathrm{C}(\bullet), 40^{\circ} \mathrm{C}(\Delta)$. Linhas de amarração: $20^{\circ} \mathrm{C}(-), 40^{\circ} \mathrm{C}(---)$.

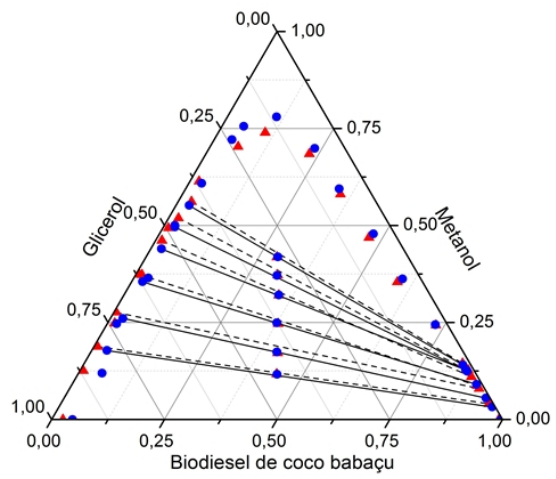


Observa-se que a solubilidade de metanol é maior na fase rica em glicerol, ou seja, sua concentração é maior nesta fase, o que pode ser justificado pela possível formação de ligações de hidrogênio entre os grupos hidroxila de ambos componentes. A partir da análise da Figura 1 , pode-se constatar que o efeito da temperatura pode ser negligenciado.

\subsection{Coeficiente de Distribuição e de Seletividade}

Um importante parâmetro estudado na extração líquido-líquido é o coeficiente de distribuição $(\beta)$, que denota como o componente se distribui nas fases de estudo Também foi determinado o parâmetro seletividade $(\mathrm{S})$, que indica o potencial de extração do solvente (Sandler, 2006). A Tabela 3 apresenta os valores dos coeficientes de distribuição $(\beta)$ e seletividade (S) para o sistema biodiesel de coco babaçu (1) + metanol (2) + glicerol (3), a 20 ${ }^{\circ} \mathrm{C}$ e $40{ }^{\circ} \mathrm{C}$.

Tabela 3 - Coeficientes de distribuição do glicerol $\left(\beta_{1}\right)$, do metanol $\left(\beta_{2}\right)$ e a seletividades (S) para o sistema biodiesel de babaçu (1) + metanol (2) + glicerina (3), a 20 e $40^{\circ} \mathrm{C}$.

\begin{tabular}{ccc}
\hline \multicolumn{3}{c}{ Biodiesel de babaçu + metanol + glicerol a $20^{\circ} \mathrm{C}$} \\
\hline$\beta_{1}$ & $\beta_{2}$ & $\mathrm{~S}$ \\
\hline$\left(\mathrm{w}_{1}{ }^{\mathrm{I}} / \mathrm{w}_{1}{ }^{\mathrm{II}}\right)$ & $\left(\mathrm{w}_{2}{ }^{\mathrm{I}} / \mathrm{w}_{2}{ }^{\mathrm{II}}\right)$ & $\left(\beta_{2} / \beta_{1}\right)$ \\
\hline 0,0201 & 0,2553 & 12,70 \\
0,0299 & 0,2866 & 9,59 \\
0,0126 & 0,2126 & 16,93 \\
0,0084 & 0,1817 & 21,73 \\
0,0331 & 0,2503 & 7,55 \\
\hline
\end{tabular}

\begin{tabular}{ccc}
\hline \multicolumn{3}{c}{ Biodiesel de babaçu + metanol + glicerol a $40^{\circ} \mathrm{C}$} \\
\hline$\beta_{1}$ & $\beta_{2}$ & $\mathrm{~S}$ \\
\hline$\left(\mathrm{w}_{1} / \mathrm{w}_{1}{ }^{\mathrm{II}}\right)$ & $\left(\mathrm{w}_{2}{ }^{\mathrm{I}} / \mathrm{w}_{2}{ }^{\mathrm{II}}\right)$ & $\left(\beta_{2} / \beta_{1}\right)$ \\
\hline 0,1411 & 0,2334 & 1,65 \\
0,2099 & 0,2388 & 1,14 \\
0,1111 & 0,2889 & 2,60 \\
0,0506 & 0,2165 & 4,28 \\
0,2759 & 0,2439 & 0,88 \\
\hline
\end{tabular}

Os baixos valores dos coeficientes de distribuição do metanol, obtidos para ambos os sistemas em estudo, confirmam que este componente se distribui preferencialmente na fase rica em glicerol. A seletividade, para ambos os sistemas apresentou valores superiores a 1, confirmando a boa capacidade extrativa por parte do solvente (glicerol).

\subsection{Confiabilidade dos Dados Experimentais}

Para o estudo da confiabilidade dos dados experimentais, utilizou-se a correlação de Hand. A Tabela 4 mostra as correlações dos dados experimentais deste trabalho. Os ajustes determinados para a correlação de Hand são próximos à unidade, o que resulta na confiabilidade dos dados experimentais.

Tabela 4 - Valores A, B e R² das correlações de Hand para os sistemas biodiesel de babaçu (1) + metanol (2) + glicerol (3) a 20 e $40{ }^{\circ} \mathrm{C}$

\begin{tabular}{ccccc}
\hline & \multicolumn{3}{c}{ Correlação de Hand } \\
\hline Temperatura & A & B & $\mathrm{R}^{2}$ \\
\hline & Biodiesel de babaçu + metanol + glicerol & & \\
\hline $20^{\circ} \mathrm{C}$ & 1,07894 & 1,27411 & 0,98821 \\
$40^{\circ} \mathrm{C}$ & 1,36337 & 1,40629 & 0,98243 \\
\hline
\end{tabular}




\subsection{Modelagem Termodinâmica: NRTL, UNIQUAC, UNIFAC}

Os modelos NRTL, UNIQUAC e UNIFAC foram utilizados para correlacionar os dados experimentais dos sistemas de biodiesel de coco babaçu + metanol + glicerol a $20{ }^{\circ} \mathrm{C}$ e $40^{\circ} \mathrm{C}$. A Tabela 5 apresenta os parâmetros de interação binária determinados para os modelos NRTL e UNIQUAC. As Figuras 2 e 3 apresentam os gráficos comparativos entre os modelos termodinâmicos aplicados e os dados experimentais. A Tabela 6 mostra matematicamente os desvios médios de cada modelo quando relacionados aos dados experimentais.

Tabela 5 - Parâmetros de interação NRTL e UNIQUAC entre biodiesel (1), metanol (2) e glicerol (3) a 20 e 40 ${ }^{\circ} \mathrm{C}$

\begin{tabular}{|c|c|c|c|c|c|c|c|c|}
\hline \multirow[t]{3}{*}{ Par i j } & \multicolumn{8}{|c|}{ Modelagem termodinâmica } \\
\hline & \multicolumn{4}{|c|}{ NRTL } & \multicolumn{4}{|c|}{ UNIQUAC } \\
\hline & Aij & Aji & $\operatorname{Bij}(1 / K)$ & $\operatorname{Bij}(1 / K)$ & Aij & Aji & $\operatorname{Bij}(1 / K)$ & $\operatorname{Bij}(1 / K)$ \\
\hline 32 & $-493,32$ & 12477 & $-1,5188$ & $-34,006$ & 428,98 & $-460,09$ & $-2,6710$ & 4,1425 \\
\hline 31 & $-1304,2$ & $-350,32$ & 7,0345 & 3,1221 & $-3910,0$ & $-5,6454$ & 17,416 & 9,5980 \\
\hline 21 & $-1244,4$ & 911,14 & 16,747 & $-4,2155$ & $-1193,9$ & 728,82 & 13,174 & $-3,6743$ \\
\hline
\end{tabular}

Figura 2 - Linhas de amarração experimentais (匹), calculadas pelo modelo NRTL $(\boldsymbol{\Delta})$, UNIQUAC ( - ) e UNIFAC $(*)$ a $20^{\circ} \mathrm{C}$.

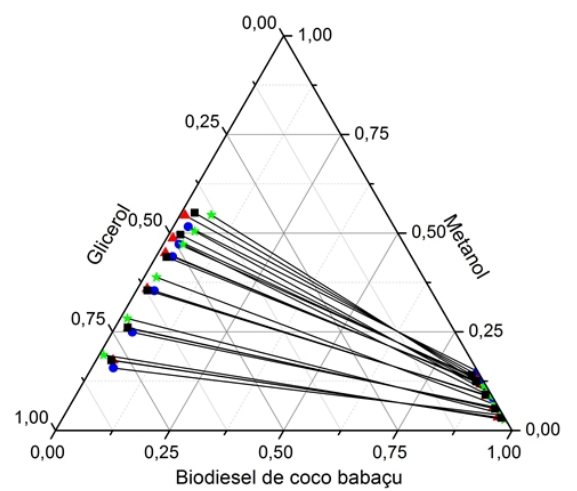

Figura 3 - Linhas de amarração experimentais (ם), calculada pelo modelo NRTL ( $\boldsymbol{\Delta})$, UNIQUAC ( - ) e UNIFAC $(*)$ a $40^{\circ} \mathrm{C}$.

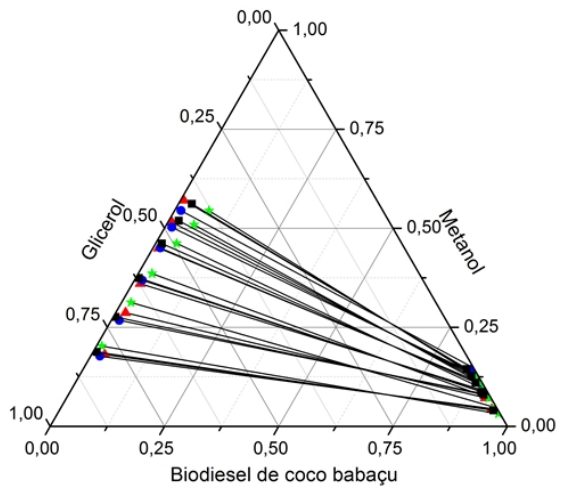

Tabela 6 - Desvio médio para modelos termodinâmicos para sistemas compostos de biodiesel de babaçu (1) + metanol (2) + glicerol (3)

\begin{tabular}{cccc}
\hline \multicolumn{5}{c}{ Modelagem termodinâmica } \\
\hline $\mathrm{T}\left({ }^{\circ} \mathrm{C}\right)$ & NRTL & UNIQUAC $-\delta_{\mathrm{x}}(\%)$ & UNIFAC $-\delta_{\mathrm{x}}(\%)$ \\
\hline 20 & 0,90210 & 1,9164 & 4,4756 \\
40 & 1,1052 & 1,3450 & 3,6057 \\
\hline \multicolumn{4}{c}{ Desvio global médio } \\
\hline \multicolumn{4}{c}{1,0088} \\
\hline
\end{tabular}

\section{CONCLUSÃO}

Tendo como base os dados obtidos nos experimentos realizados com equilíbrio líquidolíquido do sistema biodiesel de coco babaçu + metanol + glicerol, verificou-se pouca 


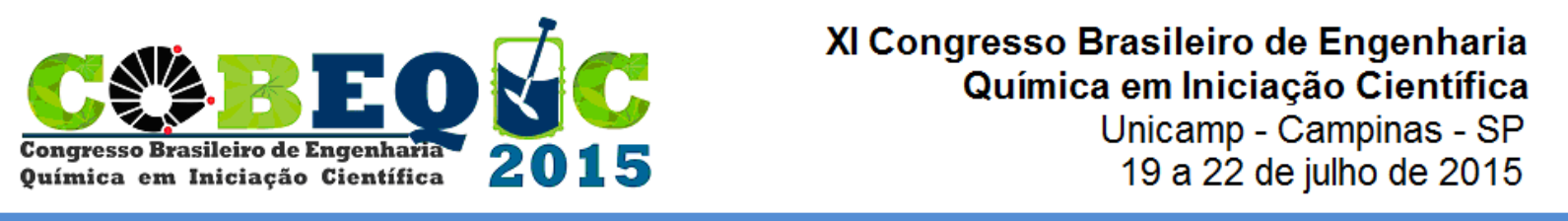

influência da temperatura na solubilidade dos componentes. A confiabilidade, testada usando a correlação de Hand, apresentou coeficientes de regressão próximos da unidade, comprovando a qualidade dos dados experimentais. Os coeficientes de distribuição apresentaram baixos valores, mostrando que o etanol distribuiu-se preferencialmente na fase rica em glicerol. A seletividade apresentou valores superiores a unidade, demonstrando que o glicerol funciona como um bom solvente para o sistema estudado. A modelagem termodinâmica realizada com os modelos UNIQUAC, NRTL e UNIFAC correlacionou os dados experimentais dos sistemas estudados e revelou valores de desvio médio satisfatórios, uma vez que variaram de $1,0088 \%$ a 4,0640 \%.

\section{REFERÊNCIAS}

ABRAMS, D. S.; PRAUSNITZ, J. M. Statistical Thermodynamics of Liquid Mixtures: A New Expression for the Excess Gibbs Energy of Partly or Completely Miscible Substances, AIChE J., v. 21, p. 116-128, 1975.

FREDENSLUND, A. A.; GMEHLING, J.; RASMUSSEN, P. Vapour-Liquid Equilibria using UNIFAC, Elsevier, Amsterdam, 1977.

GONZALEZ, J.R.A.; MACEDO, E. A.; SOARES, M.E.; MEDINA, A.G. Liquid-liquid equilibria for ternary systems of water-phenol and solvents: data and representation withmodels. Fluid Phase Equilibria, v. 26, p. 289-302, 1986.

LIMA, J.R.O.; SILVA, R.B.; SILVA, C.C.M.; SANTOS, L.S.S.; SANTOS JR, J. R.; MOURA, E. M.; MOURA, C. V. R. Biodiesel de babaçu (orbignya sp.) obtido por via etanólica. Química Nova, v. 30, p. 600 - 603, 2007.

MAGNUSSEN, T. UNIFAC parameter table for predction of liquid-liquid equilibria. Ind Eng Chem Process Des Dev, v. 20, p.331-9, 1981.

NELDER, J. A.; MEAD, R. A. A simplex method for function minimization. Comp. J., v.7, n. 4, p. 308-313, 1965.

KNOTHE, G.; Van GERPEN, J. H.; Manual do Biodiesel. 2. ed., AOCS Publicações, Urbana, 2008.

RENON, H; PRAUSNITZ, J. M. Local Compositions in Thermodynamics: Excess Functions for Liquid Mixtures, AIChE J., v. 14, p. 135-144, 1968.

SANDLER, S. I. Chemical, biochemical, and engineering thermodynamics. New York: John Wiley \& Sons Inc., 2006. 960 p.

STRAGEVITCH, L.; d'AVILA, S. G. Aplication of a Generalised Maxium Likelihood Method in the Reduction of Multicomponent Liquid-Liquid Equilibrium Data. Braz. J. of Chem. Eng., v. 14, p. 41-52, 1997.

ZHANG, Z.; HILL, G. A. Ternary Liquid-Liquid Equilibria of Water, Ethanol, and Oleic Acid. J. Chem. Eng. Data, v. 36, p. 453- 456, 1991. 